\title{
LA NAVEGACIÓ DE CONSERVA A L’EDAT MITJANA
}

\author{
Maria Teresa Ferrer i Mallol \\ Institució Mild $i$ Fontanals (CSIC) $i$ \\ Institut d'Estudis Catalans
}

\begin{abstract}
SUMARI
1. La navegació de conserva a la flota mercant.- 2. La navegació de conserva a la flota de guerra.
\end{abstract}

\section{La NAVEgació de CONSERVA a la Flota MERCANT}

En temps de guerra o bé en els períodes en que se sabia que hi havia corsaris i pirates hostils a la mar, la flota mercant prenia precaucions per tal d'afrontar el perill. En primer lloc, els vaixells solien embarcar més armes $i$ alguns ballesters per tal de poder-se defensar en cas d'ésser atacats $i$, en segon lloc, arribaven a acords de navegació conjunta entre dues naus o més per tal de poder-se ajudar mútuament si trobaven enemics. Aquests acords de navegació conjunta podien ésser adoptats de manera lliure pels interessats, pero sovint eren les autoritats competents les que obligaven els vaixells mercants a navegar de conserva.

A la costa catalana, tenim constància d'algunes disposicions generals d'aquesta mena. Les primeres que coneixem per ara són les ordinacions marítimes del 1331, promulgades per Alfons el Benigne per causa de 
la guerra contra Genova, que imposaven l'armament dels vaixells, la navegació de conserva i altres precaucions semblants ${ }^{1}$.

\begin{abstract}
Item, que nenguna nau no vaia en ultramar, que no sia de port de .III. millia salmes ensús, e que sia de .III. cubertes, e que en les dites parts tota hora vagen.II. naus en conserva, anant e tomant del port damunt dit o de maior.

Emperò tota hora entenen que $n$ qualque part que vagen o venguen les dites naus, vagen de .Iles. en lles. en conserva, ho més, mas tota hora vagen dues, e que la conserva dur, si venen de les parts de Sicília e de Nàpols o d'altres, axí com posat és, que quant seran en les ylles de Mallorche que's puguen partir, si-s volran, per venir en les parts dessa. E assò entenen d'aquelles qui van d'assí en Scicília o en aquelles parts damunt dites. $E$ assò posam pena de $\mathrm{D}$ libras, de la una a l'altre part, que pus que la conserva sia fetaa e promesa, e que's dege fer ab carta, e que la un dege defendre l'altra. E aquell qui trencaria la dita conserva, pac les dites .D. libras, e encara que la persona estiga a mercè del senyor rey d'aquell qui la conserva trencarà. Et d'assò entenem de naus o de lenys o altres vaxells qui conserva se prometen. $E$ en assò no sia entès naus o coches qui venguen per esta mar e en les ylles de Malorche e fins en Flandes.
\end{abstract}

Les ordinacions del 1356, motivades per una nova guerra amb Gènova, no obligaven a la navegació de conserva, en contrast amb les del 1331. Tots els vaixells, pero, llevat del petit tràfic de cabotatge per la costa catalana, amb mercaderies de poc valor, havien de demanar llicència abans de sortir de port; les ordinacions exigien vaixells de tonatge gros o mitjà per a les rutes llargues o mitjanes $i$, a més, un nombre adequat d'homes per a poder resistir, si eren atacats. Navegar o no de conserva devia ésser decisió privada. El batlle general $\mathrm{i}$ el vice-almirall de Catalunya vigilaven el compliment d'aquestes normes de navegacio, les destinacions i que els vaixells no portessin mercaderies de treta vedada. Per voler del rei, des del mes de febrer del 1357, els consellers de Barcelona tingueren l'última paraula en l'atorgament o no de les llicències dels vaixells que sortien de la ciutat, ja que hi havia hagut dubtes i discussions sobre la interpretacio de

' J. M. MAdurell, Ordenanzas maríimas de 1331 y 1333, "Anuario de Historia del Derecho Español", XXXI (1961), pp. 611-628. 
les ordinacions reials, especialment pel que feia al tonatge dels vaixells ${ }^{2}$. En el segle XV els consellers de Barcelona pogueren, a més, dictar ordinacions sobre la navegacio; per exemple, el 1458 disposaren que cap vaixell no sortís de Barcelona cap a Llevant o cap a Ponent si no navegava de conserva amb un altre i si no prestava les seguretats corresponents als consols de la mar $^{3}$. Els consellers $i$ els consols envaïren així un terreny que abans corresponia al batlle general i que només compartien en determinades ocasions, com la ja esmentada del $1357^{4}$.

Fins i tot quan les autoritats imposaven l'obligació de navegar de conserva, no descendien al detall d'organitzar els combois, almenys pel que sabem per ara, com veurem que era l'estil de Genova. Al llibre de llicències de navegacio de 1357-1358, almenys, no hi he trobat ordres de navegar de conservas. Ignorem si els consols de la mar podien organitzar combois per a la navegació de conserva, ja que a penes ens ha pervingut documentació del Consolat de Mar d'aquesta època. Sembla més aviat que els patrons decidien amb qui navegaven en comboi $\mathrm{i}$ acordaven lliurement entre ells les condicions de navegació conjunta en pactes que prenien la forma d'un contracte de societat.

El Llibre del Consolat de Mar regulà alguns aspectes dels pactes de navegació de conserva i dictaminà que, si un senyor de lleny o nau que tenia por d'ésser atacat per enemics o mala gent demanava a un altre de navegar de conserva amb ell i aquest, després de concedir-ho, no respectava l'acord, el dany que pogués sofrir el que havia fet la petició havia d'ésser esmenat pel company de conserva, perque el primer no hauria emprès la navegació si no hagués confiat en l'ajut del company. En canvi, si tots dos respectaven l'acord $i$, aixi i tot, l'un d'ells prenia dany, l'altre no estava obligat a cap esmena. Les coses canviaven una mica si qui havia rebut la peticio d'anar de conserva,percebia alguna remuneracio,per aquest motiu, del vaixell demandant de proteccio; en aquest cas, si el demandant de protecció rebia dany, el company de conserva estava obligat a esmenar

${ }^{2}$ A. DE CAPMANY Y DE MONTPAIAU, Memorias históricas sobre la marina, comercio y artes de la antigua ciudad de Barcelonca, reed. anotada a cura de E. GIRAI.T Y RAVENTÓS i C. BATLLE Y GAI.LART, II, Barcelona, 1962, docs. 169 i 177.

${ }^{3}$ Ibidem, doc. 376.

4 Sobre aquestes qüestions of. M. T. FERRER I MALLOL, Le port de Barcelone: Juridiction et contrôle de la mavigation, en curs de publicació.

s ACA, RP, Batllia general de Catalunya, classe 7, Fletes guias y cauciones, reg. 1057. 
el dany que el vaixell protegit hagués pres i la part proporcional del dany pres per la mercaderia. En quedava exclosa la indemnització si qui que s'havia obligat a donar protecció no podia dispensar-la per mal temps o força superior d'enemics. El Llibre del Consolat de Mar feia constar també que, si el senyor del vaixell acordava anar de conserva amb algú més, de manera remunerada o no, i no n'informava els mercaders, aquests darrers no estaven obligats a res respecte a l'altre vaixell $i$, si de resultes de la conserva rebien dany, havien d'ésser indemnitzats pel senyor de llur vaixell .

Aquestes previsions del Llibre del Consolat de Mar no coincideixen gaire, perd, amb les estipulacions que figuren en un contracte de societat per a navegar de conserva que he trobat. Aquesta societat fou pactada per a la durada d'un viatge de Barcelona a Caller, que s'havia d'efectuar durant l'any 1334. Recordem que aquest any era vigent la guerra entre catalans $i$ genovesos i que, per tant, les mars per on havien de navegar els contractants eren perilloses ${ }^{7}$. El contracte fou estipulat per Bernat Saera i Jaume Serra, tots dos ciutadans de Barcelona i patrons, respectivament, de la coca "Santa Eulalia" i de la coca baionesa "Santa Maria la Rosa".

En aquest cas, el contracte de conserva preveia, és clar, la navegació en comboi i la protecció mútua:

\begin{abstract}
Promitimus etiam pars nostrum parti et nobis ad invicem quod in dicto viatico tenebimus inter nos iustam, fidelem et legalem conservam ita quod altera pars nostrum ab altera parte non recedat aliquo ingenio sive fraude et quod deffendamus pars nostrum partem et nos ad invicem toto posse et viribus nostris contra omnes personas et quod super predictis omnibus et singulis erimus fideles et legales.
\end{abstract}

Pero el contracte preveia també una veritable societat entre les dues coques que emprenien un viatge tan arriscat. Tot el nolit que aconseguissin ambdues coques per a l'entrada del viatge, és a dir, per al trajecte Barcelona-Caller, s'havia de partir a mitges entre tots dos patrons $i$, el mateix repartiment, calia fer-lo amb tot el que poguessin guanyar en el viatge, al-lusió potser a possibles preses a costa dels enemics:

\footnotetext{
- Libre del Consolat de Mar, ed. a cura de G. COLON i A. GARCIA, I, Barcelona, Fundació Noguera i Fundació Salvador Vives Casajuana, 1981, pp. 98-101.

$7 \mathrm{~J}$. MUTGÉ, El consell de Barcelona en la guerra catalano-genovesa, durante el reinado de Alfonso el Benigno, "Anuario de Estudios Medievales", 2, 1965, pp. 229-256.
} 


\begin{abstract}
Et promitimus et convenimus pars nostrum parti et nobis ad invicem quod totum id quod nobis et utrique nostrum datum fuerit de naulo in introhitu dicti viatici tam de personis quam de rebus quam alias et totum id quod, volente Domino, adquiremus cum dictis cochis in dicto viatico dividemus inter nos ambos comuniter equis partibus medio per medium.
\end{abstract}

El contracte pactava una multa de 200 lliures de moneda barcelonina de tern que hauria de pagar el contractant que no observés algun dels punts acordats a l'altre soci de conserva, el qual podria cobrar integra la multa si no s'acudia a judici. Si els contractants acudien als tribunals, la meitat de la pena havia d'ésser per al rei o per al tribunal i l'altra meitat per al soci que hagués observat el pacte. Com a garantia del contracte tots dos socis obligaren els béns i juraren cumplir-lo, renunciant als costums de Barcelona que posessin algun obstacle al pagament de la multa estipulada. El contracte fou redactat en forma de carta partida per $A B C$, de manera que cadascun dels socis en pogués tenir un exemplar ${ }^{8}$.

La navegació de conserva, especialment si es tractava -com en el cas que acabem de comentar- de la forma mínima de comboi, no garantia pas la seguretat. Només era útil si calia afrontar algun vaixell isolat dedicat al cors. El mes d'agost del mateix any 1334 , quatre coques i dos llenys, que tambe navegaven de conserva des de Barcelona a Sardenya i que transportaven tropes de reforç destinades a l'illa, a més de mercaderies, foren interceptats prop de Maб per l'almirall genovès Sologrus de Nigro, que comandava deu galeres; en el combat, una de les coques i un lleny foren incendiats, una segona coca fou abandonada per por que no s'hi propagués el foc, ja que navegaven molt juntes, mentre que les altres dues foren perseguides fins que finalment es reteren. Els genovesos, amb la presa, es dirigiren al Cap de Carbonara, a Sardenya. Allà els desembarcaren els mariners catalans i retingueren només els patrons dels vaixells, els mercaders, els cavallers i els soldats, que havien de restar presoners a Genova o ésser intercanviats amb presoners genovesos. Sologrus de Nigro féu concentrar totes les mercaderies en una de les coques que, escortada per una galera armada, fou enviada a Salern, per tal de vendre-les a la fira. Les altres dues coques, amb els cavalls i les tropes capitanejades per Huguet de Cer-

${ }^{8}$ Arxiu de la Catedral de Barcelona, Notarials 228 (notari Pere de Torre), f. 36 r.-v. 
vello destinades a reforçar les guarnicions de Sardenya, foren enviades a Bonifacio?.

Cap a la darreria del segle XIV i al començament del XV, la decisio de constituir comboi armat foudecidida sovint pels interessats per afrontar moments d'inseguretat en determinades rutes marítimes. Aixo s'esdevingué, per exemple, a la línia marítima que unia Barcelona amb Síria.

L'any 1394 es formà una societat entre la galera "Santa Eulàlia", de Bartomeu Vidal, i la galera "Sant Gabriel", prestada per la ciutat de Barcelona al patro Pere de Quintana, que emprengueren viatge a Orient l'any $1395^{10}$. Malgrat que només es conserva un fragment dels pactes d'aquesta societat, hi trobem també la comunitat dels nolits, compartits per totes dues galeres. En aquesta ocasio el pacte "mig per mig" fou corregit en una quantitat de 500 florins d'or d'Arago a favor de Bartomeu Vidal per "calament" de galera, és a dir, pel desgast del vaixell, que en el cas de Bartomeu Vidal corria al seu càrrec perque la "Santa Eulalia" era seva, mentre que en el de Pere de Quintana el pes requeia en la ciutat de Barcelona, propietària de la "Sant Gabriel". Una particio "mig per mig" hauria estat, doncs, injusta.

Que con les dites galeas degen anar carragades de mercaderies, Déu volent, a les parts de ultramr, que tots los nòlits qui de les dites galeas exiran, entrant $e$ ixent, se partesquen entre los dits patrons per equals parts. Entès emperò que lo dit en Pere de Quintana hage a donar de la sua part al dit en Barthomeu Vidal per calament e per risch de la dita galea

9 F. C. CASULA, Carte reali diplomatiche di Alfonso III il Benigno, re d'Aragona, riguardanti l'Italia, CODOIN ACA, XLIV, Pàdua, 1971, doc. 428. La carta no té data però les cròniques genoveses situen un combat entre Sologrus de Nigro i vaixells catalans l'any 1334: J. MUTGE, El Consell de Barcelona en la guerra catalano-genovesa, p. 244. Altres documents també ajuden a datar-la: la carta 275 de la mateix volum de F. C. Casula, datada el 21 d'agost de 1334, es refereix a l'anada de Romeu de Corbera "qui era en les naus" i que anava prop del rei per informar-lo d'alguns pactes fets entre ells i els genovesos segons els quals podrien sortir de presó: sembla que Romeu de Corbera fou l'encarregat de negociar el rescat dels presoners. El 22 d'agost de 1334, el rei Alfons demanava el consell de la ciutat de Barcelona davant els rumors que li havien arribat d'aquesta captura: J. MUTGÉ I VIVES, La guerra contra Gènova de 1330-1335: Documentació barcelonina, "Miscel-lània de Textos Medievals", 6 (1992), doc. 41. Una informació molt completa sobre aquest combat es troba a la tesi doctoral inèdita de J. F. CABESTANY I FORT, Alfonso el Benigno y el gobiemo catalano-aragonés en la isla de Cerdeña (1327-1336), pp. 357-359: segons aquest autor, la batalla es desenvolupd entre els dies 5 i 8 d'agost de 1334.

${ }^{10}$ AHCB, IX Contractes, 7. Companyies, plec del s. XV, cit. i comentat per A. GARCIA I SANZ i N. COLL JULA + , Galeres mercants catalanes dels segles XIV $i$ XV, Bancelona, Fundació Noguera, 1994, pp. 228-230 i 280. 
appellada Santa Eulàlia sinchcents florins d'or d'Aragó, dels quals pachla meytat ans que partesquen d'ací, del nòlits qui ací se pagaran. E l'altre meytat a Barut dels nòlits qui là se pagaran. E és convengut entre los dits patrons que cascun d'ells se hage a parar a les messions de la galea de la qual és patró.

Tenim notícia d'altres viatges de conserva en la mateixa ruta d'Orient. Sembla que és el cas de quatre galeres que hi anaren l'any 1396, quatre que ho feren l'any 1397, quatre naus l'any 1401, dues galeres l'any 1409 , quatre naus el 1411, tres el 1417, cap a Rodes, i tres també el $1421^{11}$.

Les disposicions defensives eren similars a altres ports del Mediterrani. A Genova, l'Officium Gazarie i després l'Officium Maris s'ocuparen també del control de la navegacio, com ho feien a Catalunya el batlle general i el vice-almirall. A la sortida del port ligur els homes d'aquelles institucions inspeccionaven els vaixells a fi que portessin l'armament i la tripulació necessaris per a llur defensa i a més, podien imposar, si ho creien necessari, la navegacio de conserva. En aquests casos l'Officium nomenava un dels patrons de les naus afectades patró de l'expedicio, amb poders sobre les altres naus que haguessin de navegar amb ell. A vegades l'Officium ordenava a algunes naus que n'esperessin d'altres en determinats ports per a navegar després de conserva; les despeses que ocasionava l'organitzacio de la conserva, demores als ports etc., havien d'ésser distribuïdes entre els armadors i els mercaders.

$\mathrm{Si}$ al litoral catala les hostilitats entre genovesos i catalans entre els anys 1330-1336 havien determinat la imposicio de la navegacio de conserva, l'any 1330 l'Officium Gazarie exigf també el mateix requisit a tots els vaixells genovesos que anessin a Romania o a l'Occident més enllà de Mallorca $^{12}$.

"C. CARRERE, Barcelona 1380-1462. Un centre econdmic en apoca de crisi, Barcelona, Curial, 1977, pp. 308-309.

12 V. VITALE, Le fonti del Diritto Marittimo Ligure, a Fonti del Diritto Marittimo Italiano, I, Gènova, 1959, pp. 103, 105 i ss., 165-167. 
Els registres de l'Officium Maris, que vaig estudiar fa temps, també apleguen disposicions per a la navegació de conserva d'alguns vaixells. El registre de 1402-1403 recull una ordre del mes d'agost destinada a Giuliano Centurione i a Giacomo Cappa, patrons de nau. Havien de navegar de conserva fins a Barcelona o bé fins a $\mathbf{4 0}$ milles mar endins de Barcelona vers Menorca, si es desviaven cap a aquesta illa; Giuliano Centurione fou nomenat capità del comboi i ambdós patrons foren amenaçats amb una multa de 500 florins d'or si desobeïen l'ordre. En aquest cas podem suposar que els homes de l'Officium temien atacs dels corsaris catalans i castellans que interceptaven les rutes entre la penísula italiana i la península ibérica.

Aquest mateix any, pel setembre, Nicold de Lomusso i Venturino de Ratto de Portovenere, patrons de galera, reberen ordre també de navegar de conserva, sota la capitania de Nicolo de Lomusso. Havien de sortir de Genova cap a Roma i havien de passar per una zona infestada de pirates, a la costa entre Elba i Piombino, de manera que les precaucions eren necessàries. L'ordre preveia que naveguessin de conserva des de Porto Pisano fins a Roma, sota pena de 200 florins. Com que sembla que Venturino de Ratto no podia salpar perque la xurma del seu vaixell no estava a punt, l'Officium ordenà a Nicold de Lomusso que l'esperés entre Portovenere i Porto Pisano durant dos dies; passat aquest temps, si el seu company no havia comparegut, Nicolo de Lomusso podria navegar tot sol cap a la seva destinaci $6^{13}$.

El registre de l'Officium Maris de 1408-1410 conté més ordres als vaixells mercants per a prendre precaucions defensives que el de 1402-1403 perque la hostilitat entre catalans i genovesos havia anat creixent fins a convertir-se en una guerra no declarada. El 7 de juny de 1408, l'Officium envià cartes als consols i mercaders genovesos a Sevilla $\mathrm{i}$ als massers $\mathrm{i}$ mercaders genovesos a Bruges i Londres per advertir-los del perill d'un armament de naus a Catalunya -que tenia com a objectiu perseguir el corsari Pedro de Larraondo o Larranda ${ }^{14}$ - i per pregar-los que vigilessin que les

${ }^{13}$ M. T. FERRER I MALLOL, Dos registres de l' "Officium Maris" de Gènova (1402-1403, 1408-1410), "Atti del I Congresso Storico Liguria-Catalogna (1969), Bordighera, Istituto Internazionale di Studi Liguri. 1974, pp. 252-253 i docs. 3 i 4.

14 Sobre aquest armament ef. M. T. FERRER I MALLOL, Una flotta catalana contro $i$ corsari nel Levante (1406-1409), en curs de publicació en el volum en homenatge al Prof. Geo Pistarino. 
naus genoveses anessin ben proveïdes d'homes i d'armes, que les fessin navegar de conserva i que, si calia, imposessin penes i retinguessin els vaixells. Cartes semblants foren enviades el 3 d'agost als consolats de Napols i de Gaeta pels armaments que es feien a Sićlia amb el mateix objectiu de perseguir Pedro de Larraondo, ordres que foren repetides el 3 de setembre del mateix any. L'any 1409 Pietro da Voltaggio, Giovanni Ghisolfi, Enrico Lecavelli i Giovanni Badinella reberen ordres per navegar de conserva des de Messina cap a Orient; igualment en reberen Damiano de Camilla, Pietro Antonio di Lagneto i Antonio da Bavari per al trajecte de Gènova a Gaeta; Giacomo Marchesano, Nicolo da Levanto i Simone Bairo, que havien d'anar cap a Occident, i Paolo Italiano, Montanino Burnengo i Lanzarotto da Borzoli, que anaven a Sicflia.

Durant l'any 1410, el registre de l'Officium Maris ens informa de l'organització d'una conserva o comboi per a la nau d'Angelo de Negro, que anava cap a Nàpols i Romania. Havia d'anar acompanyada per les naus de Paganino de Biassa, Enrico Lecavelli, Giovanni di Ghisolfi o be Battista Ardimento. Cap a Occident es formà un comboi amb les naus de Paolo Italiano i Megolo Lercari, pero hi devia haver problemes per a la sincronització del viatge perque primer hom ordenà a Paolo Italiano que esperés Megolo Lercari a Cadis; després, aquesta ordre de conserva fou revocada i substituïda per una circular als patrons genovesos de naus de més de $\mathbf{7 0 0}$ bótes que anaven cap a Occident, en la qual hom els manava que naveguessin de conserva amb Paolo Italiano i Megolo Lercari. També aquesta nova ordre fou revocada perque fou expedida una carta patent a tots els patrons genovesos que es dirigien cap a Occident, la qual els manava navegar de conserva des de Savona. El motiu d'aquestes precaucions era la presencia al Mediterrani de quatre naus armades catalanes que, segons l'Officium, anaven en cors contra genovesos ${ }^{15}$.

\section{La NAVEgació de CONSERVA a La flota DE GUERRA}

L'associació de conserva es practicava també entre embarcacions armades dedicades al cors o a la policia maritima. Un exemple molt clar n'és l'acord pactat l'any 1315 entre la flota dita de l'Almoina, integrada

is Ibldem, pp. 254-259 i docs. 11, 13-16, 22-23, 25-29 i 34. 
pels contingents de les ciutats marítimes catalanes $i$ valencianes, $i$ un petit estol castella, armat per l'arquebisbe i la ciutat de Sevilla i per l'almirall de Castella, Alfonso Jofre de Loaysa. L'estol castellà estava format per quatre galeres, un lleny, una nau i una barca; el nombre de vaixells de l'armada catalana no s'hi especifica, però devia ésser força superior, al voltant de les tres cinquenes parts del conjunt; era comandada pel capità del contingent de Barcelona, Ramon Ricart, i pel capità del de Valencia, Bartomeu Matoses. Sembla que totes dues flotes, que tenien l'objectiu comú de netejar la mar de corsaris i pirates sarraïns, es trobaren en mar $i$ decidiren navegar de conserva durant vint dies; l'acord més important fou que durant aquest període de temps tot allo que prenguessin a moros, tots junts o per separat, es repartiria proporcionalment al nombre de persones de cada armada:

E que los dichos capitanes fizieran su conserva buena e verdadera con el dicho Alfonso Jofre por XX días, en tal manera que todas las cosas que Dios les diesse a ganar de los moros a todos en uno e cadauno por sí, en mar o en tierra, en los dichos XX días que partiessen egualmente por cabeças e por nombres de personas.

La flota catalano-valenciana féu la reserva que no podia atacar ni prendre res dels reis de Tunis i de Bugia ni de llurs súbdits, perquè estaven en treva amb la Corona d'Aragó.

No conservem el document del pacte, que fou escrit i jurat amb prestació d'homenatge per part dels contractants, pero se'n conserva una àmplia referència a través d'una carta del rei Jaume II a les autoritats de Sevilla, que havien protestat pel repartiment del botf. Efectivament, durant el perfode de vigència de la conserva, la flota conjunta prengué una nau procedent de Tunis que portava molts moros i mores i joies de gran valor; pero el patró de la nau capturada declarà que els moros i els béns pertanyien a un germà del rei de Tremissèn, el qual, veient que la nau seria presa per la flota catalano-castellana, havia fugit amb una barca cap a la costa proxima. Per tant els catalans i valencians exigiren llur part del botl, les tres cinquenes parts, que pujaren a 5.000 dobles d'or. Els castellans pretenien que els moros $i$ els béns eren de Tunis perque així creien que podrien adjudicar-se gairebé tot el botf, ja que els catalans no se'l podien quedar perque tenien treva amb Tunis. Per aixo les autoritats sevillanes consultaren el rei Jaume II per coneixer la seva versió, que era la del capità barcelonf, Ramon Ricart, i la que acabem de resumir. A la seva resposta el rei aclaria que, si els moros presos haguessin estat súbdits del rei de Tunis, no sola- 
ment la seva flota no hauria participat en el botí sino que hauria hagut d'impedir que l'estol castellà els el prengués; quedava clar, doncs, que el botí era legal per a la Corona d'Arag $\sigma^{16}$. Els consellers de Barcelona també escriviren als alcaldes i a l'algutzir de Sevilla pel mateix motiu pocs dies després que ho fes el rei ${ }^{17}$

Com a conclusio, podem dir que la navegació de conserva era, durant l'Edat Mitjana, una precaució defensiva per a la marina mercant en èpoques conflictives, però era també una possibilitat de coordinació ofensiva en la marina de guerra. Quan enemics o corsaris solcaven les mars, les autoritats que regulaven la navegacio, tant a Catalunya com a Gènova, ordenaren que els vaixells mercants naveguessin de conserva, formant comboi. Aquesta coordinació d'un o més vaixells prenia, a efectes legals, la forma d'un contracte de societat, valid per a un viatge o per a un període de temps determinat.

\section{RÉSUMÉ}

La navigation de conserve était, pendant le Moyen Age, une précaution défensive pour la marine marchande en époques conflictives, mais était aussi une possibilité de coordination offensive pour la marine de guerre. Quand ennemis ou corsaires sillonnaient la mer, les autorités qui réglaient la navigation, tant dans la Catalogne comme à Gênes, ordonnèrent que les bateaux marchands navigassent de conserve, en formant comboi. Cette coordination, dans le champ légal, la forme un contrat de société, valide pour un voyage ou une période determinée de temps.

${ }^{16}$ ACA, C, reg. 242, ff. 261 v. -262 v. i AHCB, Borradors de lletres, I-1, núm. 2 (1315, octubre, 26).

${ }^{17}$ A. de Capmany, Memorias históricas, II, doc. 99 (1315, novembre, 6). 


\section{SUMMARY}

Protective navigation was, during the Middle Ages, a defensive precaution taken by merchant shipping in times of conflict, but it was also a tactical possibility for warships. When enemies or pirates threatened the seas, the authorities who controlled shipping, both in Catalonia or in Genoa, ordered merchant vessels to navigate protectively, forming a convoy. This cooperation with one or more ships took on the form of a contract of corporation, valid for a voyage or for a fixed period. 